\title{
FIVE PHASE PENTAGON HYBRID STEPPER MOTOR INTELLIGENT HALF/FULL DRIVER
}

\author{
Alexandru MORAR \\ "PetruMaior" University of Târgu - Mureş, Romania \\ Nicolae Iorga st., no. 1, Tirgu-Mures, 540088, Romania \\ alexandru. morar@ing.upm.ro
}

\begin{abstract}
Stepper motors are very well suited for positioning applications since they can achieve very good positional accuracy without complicated feedback loops associated with servo systems. In this paper, an intelligent five-phase stepper motor driver of business card size proposed. Constant current chopping technique was applied for the purposes of high torque, high velocity and high efficiency. The driver was designed to drive a middle-sized hybrid stepper motor with wire current rating from 0.4 to 1.5A. An up-to-dated translator of five-phase stepping motor was used to drive the gates of $\mathrm{N}$-channel MOSFET array. The resolution in full/half mode is 0.72/0.36 degrees/step. Moreover, an automatic power down circuit was used to limit the power consuming as the motor stops. Additionally, a self-testing program embedded in a 80C31-CPU (PCL838) can self-test whether the driver is normal or not. This embedded program including linear acceleration and deceleration routines also can serve as a positioning controller. The dimension of this driver is approximate 70x65x35 millimeters, which is smaller than a business card. Experimental results demonstrate that the responses of the driver can reach 60 kilo pulses per second
\end{abstract}

Keywords: hibrid pentagon stepper motor, N-channel MOSFET array, sequencer, half/full control, PWM chopper

\section{Introduction}

Stepper motors are used to obtain the motion profiles in the field of Robotics and Automation Engineering. Stepper motors are preferred in applications where high precision controls in position and velocity, is important such as in aerospace, printers, scanners, copiers, faxes, optical and magnetic disk devices, medical equipment, $\mathrm{X}-\mathrm{Y}$ plotters, CNC machines, semiconductor fabrication equipment, agricultural automation applications, textile equipment's and precision telescope positioning systems [3][4]. Moreover reliability, lack of contact, aging, mechanical ruggedness and availability of torque at zero speed are the attractive features of Stepper Motors. Among the various types of stepping motors, the hybrid stepping motor has a permanent magnet rotor and a toothed magnetic structure on both the stator and rotor, so that a permanent-magnet torque as well as reluctance torque can be generated [1]. Two - phase stepper motors are commonly used, but five-phase stepper motors have the superior characteristics of resolution, vibration and performance compared with two phases motors.
When both stepping motors are operated in half step mode, two phase motor provides a resolution of 400 steps per revolution while a five phase motor has a resolution of 1000 steps. This resolution is 2.5 times higher when compared with two phase stepper motor. The applications where high precision, low vibration and low noise is required, five-phase hybrid stepper motor can be employed compared to two-phase hybrid stepper motor. Five-phase stepper motors are superior in dynamic performance [2]. A high precise position control using five-phase hybrid stepper motor is achieved in open loop control achieves. The higher the number of phases, smaller will be positioning deviations. Microstepping is a technology that achieves low resonance, low noise operation, at extremely low speeds by controlling the flow of electric current fed to the motor coil and thereby dividing the motor's basic step angle into smaller steps. Pulse Width Modulation (PWM) technique is usually used to excite the driver to operate the motors. This paper proposes a compact five-phase pentagon stepper motor half/full driver for positioning or speed control applications. 


\section{Structure of five - phase stepping motor}

The five-phase hybrid stepper motor is developed with high resolution with no low speed resonance problems. The five-phase stepper can also be classified under permanent magnet hybrid stepper motor. The stator windings are energized in the proper sequencer to produce a rotating magnetic field which turns the rotor. The significant advantage of the five-phase stepper motor is its excellent torque retention capability at high operating speed. The motor used for implementation is a five-phase hybrid stepper motor and it is shown in figure 1 and figure 2 . The figures above show two cross-sections of a 5phase hybrid stepping motor. Hybrid stepping motors are composed primarily of two parts, the stator and the rotor. The rotor in turn is comprised of three components: rotor 1 , rotor 2 and the permanent magnet. The rotors are magnetized in the axial direction, with rotor 1 polarized north and the rotor 2 polarized south. The stator contains 10 magnet poles with small teeth, each of which is wrapped in wire to form a coil. The coil is connected to the facing magnet pole and is wound so it becomes magnetized to the small pole when current is run through it. (Running a current through a given coil magnetizes the facing poles to the same magnetism, either North Pole or South Pole.). The two facing poles form a single phase. Since there are five phases, A trough E, the motor is called a 5- phase stepping motor. There are 50 teeth on the outside of the rotor, with the teeth of rotor 1 and rotor 2 mechanically offset from each other by half a tooth pitch.

Figure 3 and figure 4 help to describe the relationship on the positions of the stator and rotor teeth when magnetized. When phase A is excited, its poles are polarized south. This attracts the teeth of rotor cup 1, which are polarized north, while repelling the teeth of rotor cup 2, which are polarized south. Therefore, the forces on the entire unit in equilibrium hold the rotor stationary. At this time, the teeth of the phase B poles, which are not excited, are misaligned with the south-polarized teeth of rotor 2 so that they are offset at $0.72^{\circ}[5][6]$.

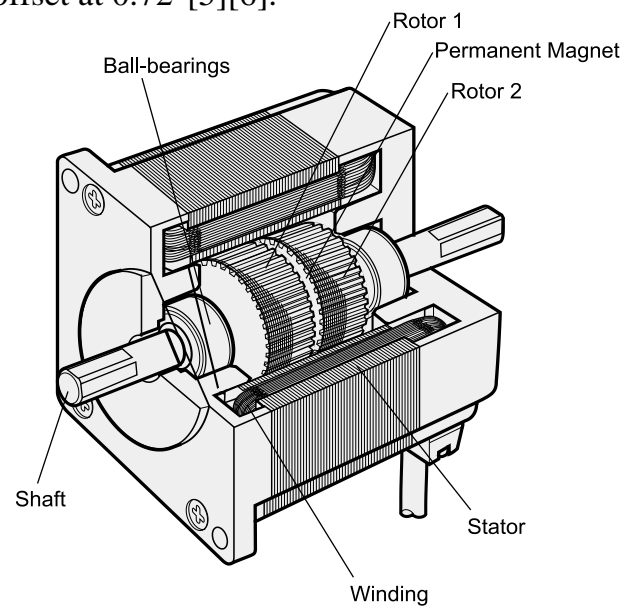

Fig. 1: 5 Phase Stepping Motor.Cross-Section Parallel to Shaft

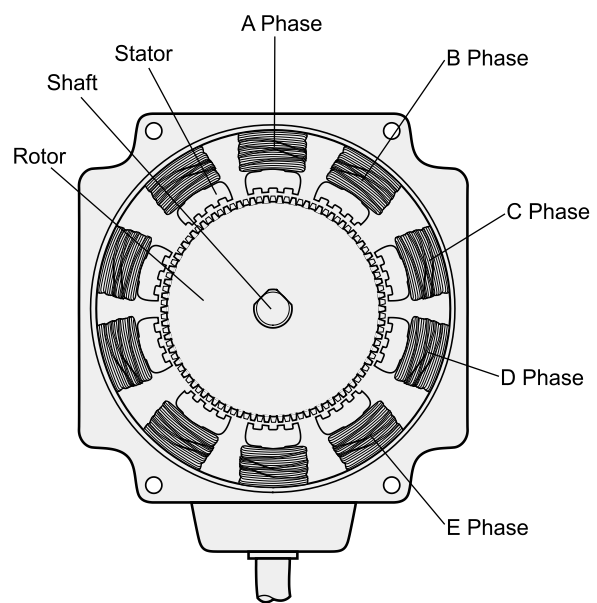

Fig. 2: 5 Phase Stepping Motor.Cross-Section Perpendicular to Shaft

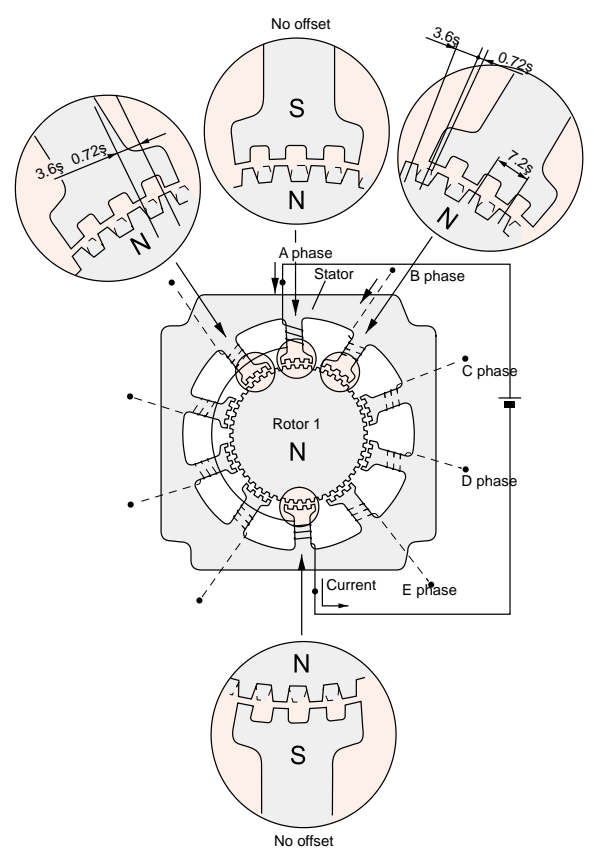

Fig. 3: The position of the stator and rotor teeth when phase $A$ is excited

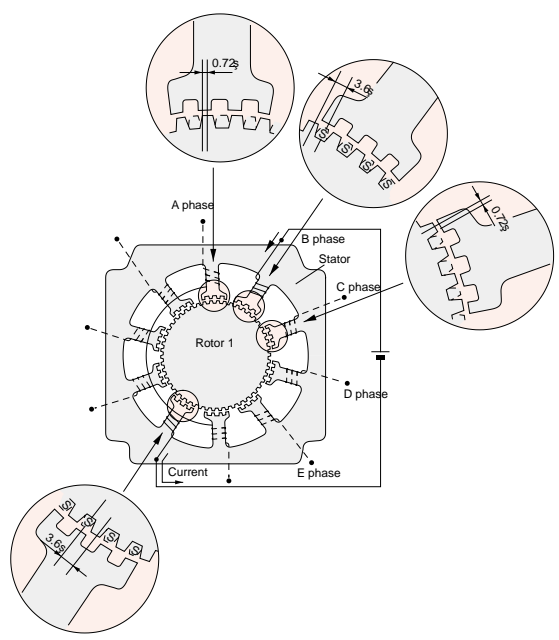

Fig. 4: The position of the stator and rotor teeth when phase $\mathrm{B}$ is excited 
When excitation switches from phase A to B shown in figure 4, the phase B poles are polarized north, attracting the south polarity of rotor 2 and repelling the north polarity of rotor cup 1 . In other words, when excitation switches from phase $A$ to $B$, the rotor rotates by $0.72^{\circ}$. As excitation shifts from phase $A$ to phases $\mathrm{B}, \mathrm{C}, \mathrm{D}$ and $\mathrm{E}$, then back around to phase $\mathrm{A}$, the stepping motor rotates precisely in $0.72^{\circ}$ steps. To rotate in reverse, the excitation sequence is reversed as phase A, E, D, C, B, then back to phase A. High resolution of $0.72^{\circ}$ is inherent in the mechanical offset between the stator and rotor, accounting for the achievement of precise positioning without the use of an encoder or other sensors [18], [19].

\section{Circuit description}

Any stepping motor driver circuit requires several logic and power circuit functions to work together to supply the proper amount of current at the right time to the stepping motor windings in order for the motor to provide accurate and smooth motion to the load.

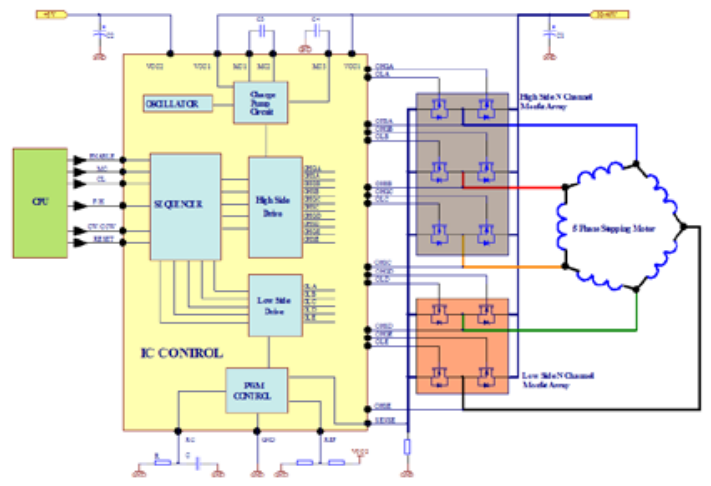

Fig. 5: Block diagram of 5-phase pentagon stepper motor driver circuit

Figure 5 above shows the basic functional block diagram of a 5-phase Pentagon stepper motor driver circuit [9][15][16]. Each of these functions is an important part of the overall circuit and close attention should be paid to each of them. The design procedure of this driver is described detailed as follows.

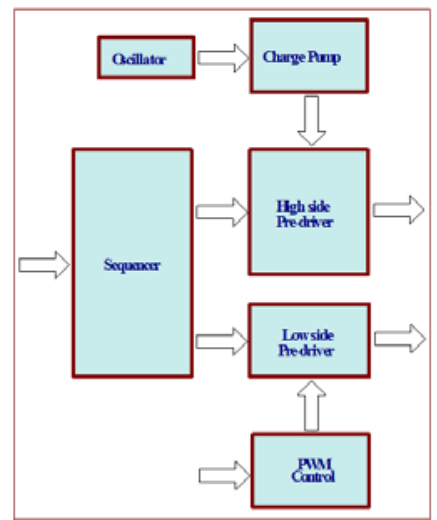

Fig. 6: The internal block diagram of SI-7510

\section{(1). Excitation sequence generator}

The excitation sequence generator function is primarily responsible for converting the step and direction input signals from the upper level controller into the proper phase sequence to cause the 5-phase stepping motor to rotate [8]. The sequence generator determines what combination of MOSFET transistors need to be turned $\mathrm{ON}$ to make the motor rotate to the next step. For a Pentagon 5-phase driver, there are 10fullstep in an electrical cycle with 4 phases being $\mathrm{ON}$ at any given time. Each full step results in $0.72^{\circ}$ of shaft rotation. Half stepping, or $0.36^{\circ} / \mathrm{step}$, is possible by alternately turning 4-phases $\mathrm{ON}$ and then 5 phases ON. The 4-5 phase ON excitation sequence has 20 steps in its electrical cycle. For compact design consideration, a five-phase stepping motor driver chip SI-7510 from Sanken electronics is adopted. SI-7510 is dedicated designed IC that imbedded a sequence circuit to control the output stage of the driver [11][12]. The internal block diagram of SI-7510 is shown in figure 6. Input and output timing chart and motor coil excitation sequence is shown in figure 7 and figure 8.Other functions that are typically included in the sequencer generator function include: Full or Half step operation selection, current Off selection, RESET of the driver, state zero(TIM) output indication.

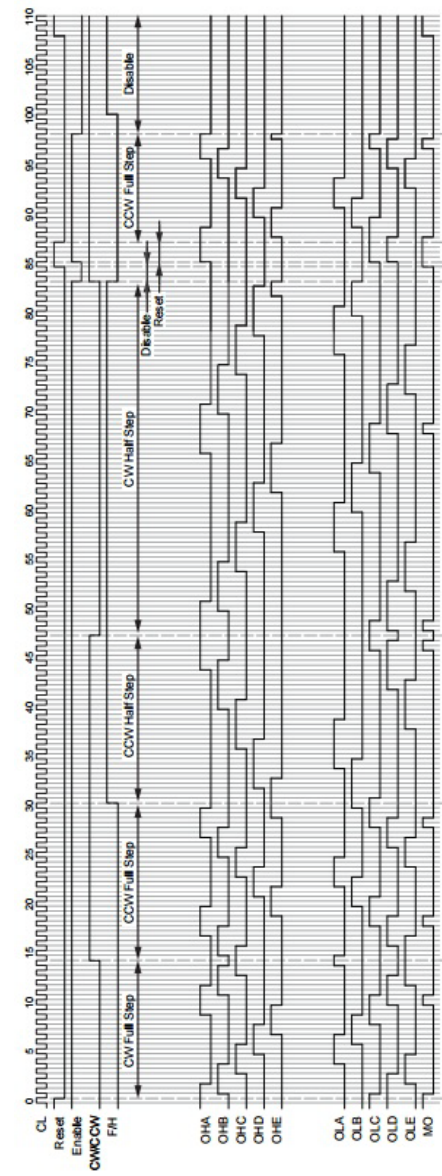

Fig. 7: Input and output timing chart of SI -7510 


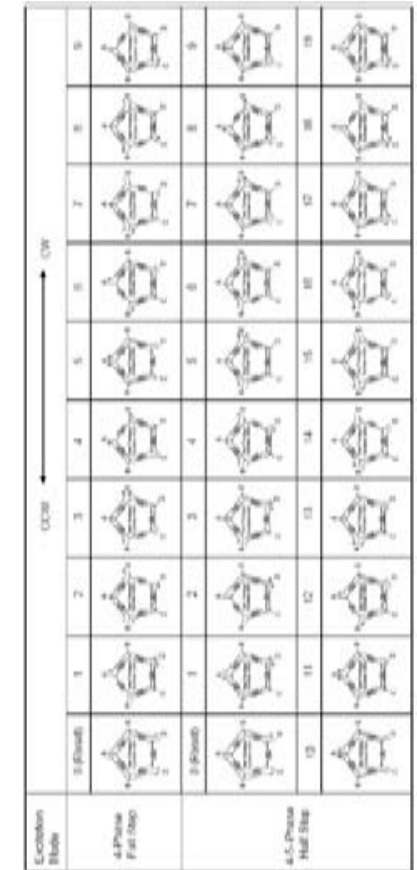

Fig. 8: Motor coil excitation sequence:4 Phase, full step, 4-5 Phase, half step

\section{(2). Change pump circuit}

The charge pump circuit is used to create a separate, floating supply voltage used by the high side pre-driver that is separate from the motor voltage. A charge pump circuit uses a number of capacitors that act as "buckets" to pump charge from one place to another. In effect, as one capacitor is charges and then switched off, the change in that capacitor is then into the next capacitor to be added to the power supply voltage. This process can be repeated as many times as you would like until the charge stored by last capacitor equals the value desired.

\section{(3). High side pre-driver}

The high side pre-driver supplies a voltage that is used for driving the MOSFET gate terminal to ensure that the MOSFET will supply the motor voltage to the winding very quickly when turned ON.

\section{(4). Low side pre-driver}

The low side pre-driver supplies a voltage that is used for driving the MOSFET gate terminal to ensure that the MOSFET will connect to ground very quickly when turned ON.

\section{(5). Motor constant current control circuit}

This circuit compares the voltage in the motor winding to a reference voltage set by the user that represents the desired current in the motor. A chopping circuit is usually used to adjust the power supply to the motor maintain a constant current in the motor winding.

\section{(6). High side N-channel MOSFET array}

The drain terminals of these MOSFETs are connected to the + power to be supplied to the motor winding and determine where the voltage will enter the windings.

\section{(7). Low side N-channel MOSFET array}

The source terminals of these MOSFETs are tied to GND and determine the path to ground that the voltage will take through the motor windings.

The output circuit diagram for 5-phase pentagon driver is shown in figure 9 below. Simply stated the 5 transistors shown in the top row determine which motor coils the voltage will enter the motor windings (high side) while the 5 transistors shown in the bottom row determine which motor coils the voltage will flow to ground through.

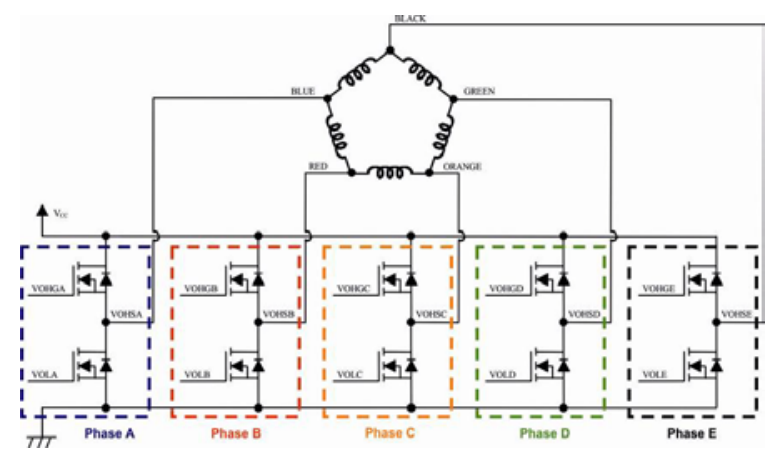

Fig. 9: Pentagon driver output circuit diagram

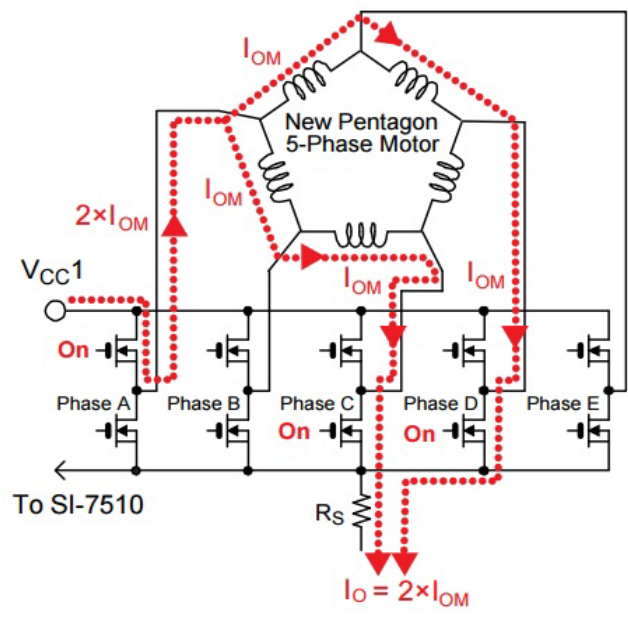

Fig. 10: Motor current path after logic reset

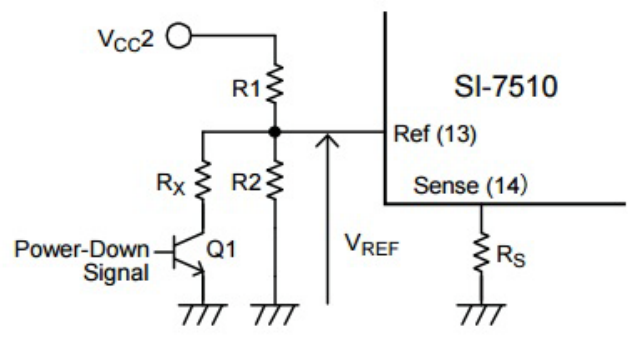

Fig. 11: Motor current setting circuit 


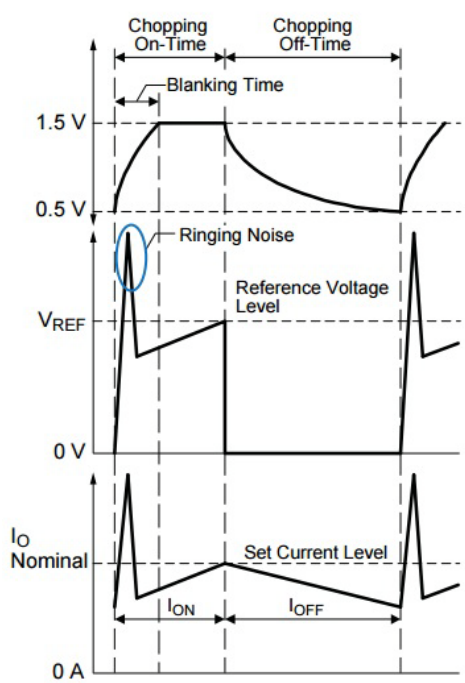

Fig. 12: Operating waveforms during chopping

\section{(8). Reset control}

Reset is non-synchronous reset function. Figure 10 show the motor current patch after the internal sequencer resets the device logic.

\section{(9). Calculating motor current}

The calculation for setting motor current, $\mathrm{I}_{0 \mathrm{M}}$, for the SI-7510 is determined by the ration of the external components R1 and R2, and the current sense resistor, $R_{s}$. The SI-7510 controls the total set current, $\mathrm{I}_{0}$ and $\mathrm{I}_{0 \mathrm{M}}$ is as follows: $\mathrm{I}_{0}=2 \times \mathrm{XI}_{0 \mathrm{M}}$. The current sense voltage, $\mathrm{V}_{\text {SENSE }}$, is generated bay $\mathrm{I}_{0}$ flowing intro the current sense resistors, $R_{S}$. The ratio of $V_{R E F}$ to $V_{\text {SENSE }}$ is $1: 1$, so $I_{0}$ can be calculated as follows(refer to figure 11): $I_{0}=V_{\mathrm{REF}} / \mathrm{R}_{\mathrm{S}}$, where $\mathrm{V}_{\mathrm{REF}}$ is calculated as: $\mathrm{V}_{\mathrm{REF}}=\mathrm{V}_{\mathrm{CC} 2} \times \mathrm{R}_{2} /\left(\mathrm{R}_{1}+\mathrm{R}_{2}\right)$.

\section{(10). Output chopping}

The operating output and feedback pins waveforms during chopping are shown in figure 12, and the current paths during chopping are shown in figure 13.

Chopping Off Time. This IC's controls output chopping off-time. Chopping off-time is determined by time constant of the external $R_{t}-C_{t}$ circuit connected to the RC terminal. The off-time is the duration for the RC terminal voltage to decrease from approximately $1.5 \mathrm{~V}$ to $0.5 \mathrm{~V}$. The chopping off-time can be calculated as: $t_{\mathrm{OFF}}=1.1 \mathrm{xR}_{\mathrm{t}} \mathrm{CC}_{\mathrm{t}}$, where $\mathrm{R}_{\mathrm{t}}$ is 15 to $75 \mathrm{k} \Omega, \mathrm{C}_{\mathrm{t}}$ is 420 to $1100 \mathrm{pF}$ (recommended value).

Blanking Time. The $\mathrm{R}_{\mathrm{t}}-\mathrm{C}_{\mathrm{t}}$ circuit time constant is also related to the blanking time, $t_{\mathrm{BRK}}$. Blanking time prevents malfunction due to ringing noise which occurs after chopping transitions from off to on. Blanking time is the duration for the RC terminal voltage to increase from approximately $0.5 \mathrm{~V}$ to 1.5 $\mathrm{V}$. When the RC terminal voltage increases to this level, a current of approximately $200 \mu \mathrm{A}$ flows out the $\mathrm{RC}$ terminal. Blanking time is calculated as:
$t_{\mathrm{BRK}}=\mathrm{C}_{\mathrm{t}} /\left(200 \times 10^{-6}\right)$. Even if the sense voltage, $\mathrm{V}_{\text {SENSE }}$, becomes higher than the reference voltage, $\mathrm{V}_{\mathrm{REF}}$, during the blanking time interval, the power control circuit does not operate and the device output always remains in the On state. Because of this, if the $\mathrm{C}_{\mathrm{t}}$ value is too large when the output current value must be set small, the output current may not fall to the set value. In addition, if the $C_{t}$ value is too small, it causes malfunction due to ringing noise.

Chopping On-Time. Chopping on-time is determined by: $\mathrm{V}_{\mathrm{CC} 1}$, the motor output time constant, and the chopping off-time.
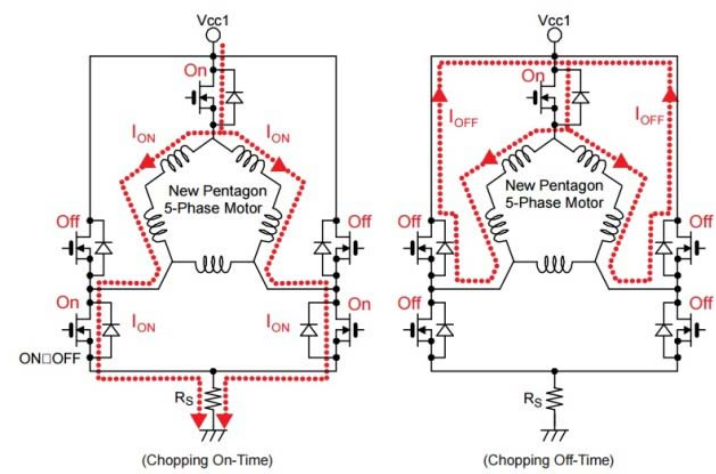

Fig. 13: Current path during chopping; dotted red line indicates path of current (left) during chopping on-time (ION), and (right) during chopping off-time (IOFF)

The configuration of the proposed driver is illustrated in figure 14.

\section{Power stage}

In the design of power stage, it dramatically increases the power consumption with bipolar transistors. Therefore, N-channel MOSFETs were selected as the output power stage to drive the windings of stepping motors. Two MOSFET chips, termed as SLA 5073(6 N-channel MOSFET array for phases A, B, C) and SLA 5074(4N-channel MOSFET array for phases D,E), were used as the power stage of the driver [7][10]. Both MOSFETs contribute to five pairs of push-pull driver to drive each coil of a five phase stepping motor. Since maximal current both SLA 5073 and SLA 5074 can bear is 5 amperes, this design can be use in most of the industrial applications. Unfortunately, because the limited dimension of the heat sink in a compact size design, the driving current of this driver is limited to 1.5 amperes. As the driving current for a motor coil is larger than 1.5 amperes, a bigger heat sink must be redesigned for better heat dissipation[16]. For a better performance while designing a constant current driver, a higher voltage applied to the output MOSFETs is preferable. However, the maximum rating of SI-7510 and MOSFET are 44volts and 60 volts, respectively. Considering the maximum rating and the capacity of heat sink, an industrial standard 24 volts is adopted as the power supply of the driver. A voltage regulator is designed in the driver to adjust 


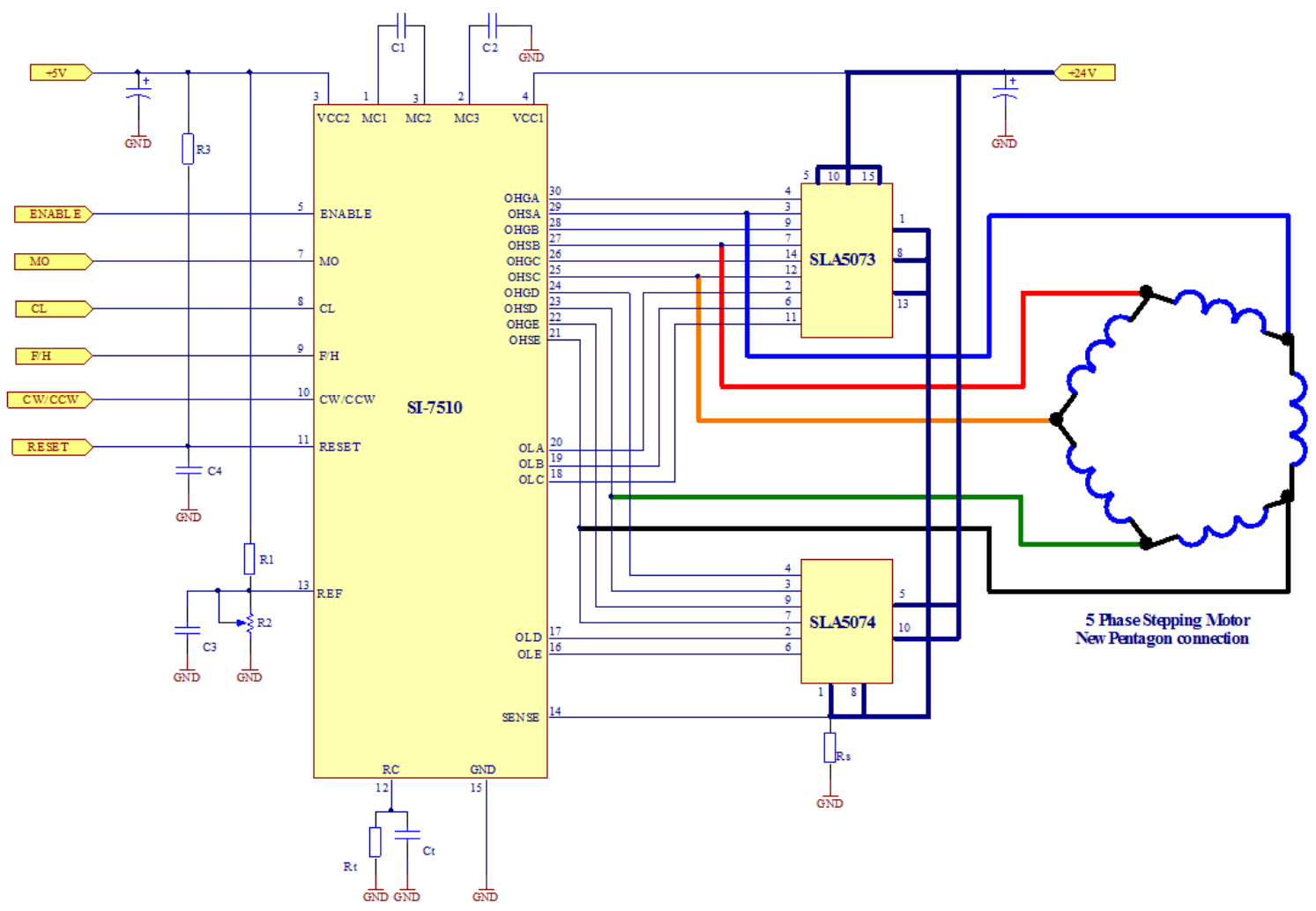

Fig. 14: Connection diagram of the proposed driver

\section{Man-machine interface}

The LED's are used as indicators for manmachine interface. Red LED indicates the power of the driver is on, green LED indicates the direction of the motor and yellow LED indicates that clock is providing by system controller. SI-7510 can operate in two different modes, four-phase excitation mode or four-five-phase excitation mode. Four-phase excitation and four-five-phase excitation mode are also termed as full step and half step mode. A five phase stepping motor in full/half step mode, can reach the resolution on 500/1000 steps per revolution, or 0.72/0.36 degrees per step. The resolution can be selected simply with a DIP switch. In a normal condition, the drivers were controlled by two signals termed as clock (CK) and direction (DIR) pulses. These two signals generated from external position control system were feed to the two input pins, CK and DIR, of the driver. However, a self-test function is preferable to test the driver whether is malfunction when the external position control system was not available. In this design, PCL-838 ISA Stepping motor control Card PC- Lab card [20] was used to provide a self-test function. The PCL- 838[20] has been specifically designed as user-friendly solution for your stepping motor control application. It's onboard 80C31-CPU, along with a $16 \mathrm{MHz}$ clock crystal, and two 8254 programmable timers, controls up to three driver axis (one motor/channel) independently and simultaneously. Digital pulse and directional output signals control each motor by step rate and clockwise/counter-clockwise (CW/CCW) direction. When the driver is operated under independent position controller mode, a linear acceleration and deceleration program was also imbedded for a smoother positioning purpose.(figure 15 shows a typical acceleration and deceleration profile). This acceleration and deceleration function can prevent the motor and mechanism from starting and stopping suddenly

\section{Results}

The dimensions of the driver are approximate 70x65x35 millimeters, which are smaller than of a business card in length and width. A positioning card (PCL-838) plugged inside an IBM PC is utilized to test the accuracy and the speed of the driver. For easy implementation, this positioning card was implemented with ISA bus interface. This card output clock and direction signals as inputs to the tested driver. A software package including several functions was designed with $C$ language so as to be feasible to test this driver. The most used function of this package was linear acceleration and deceleration so that the motor may start and stop more smoothly and allow the motor to reach a higher speed that in the case of without acceleration and deceleration. A five - phase stepping motor Model PK566-NB-A8, 
with a rated current of $1.4 \mathrm{~A}, 1.1 \Omega$, from VEXTA ORIENTAL MOTOR, Japan,was used to connect with this proposed driver. Experimental results demonstrate that the responses can reach 60 kilo pulses per second as driving a five-phase stepping motor with a phase current of 1.4 A. A 60 kilo pulses pulse rate means that the motor can rotate at speed of around 50 revolutions per second when operated in half step mode.
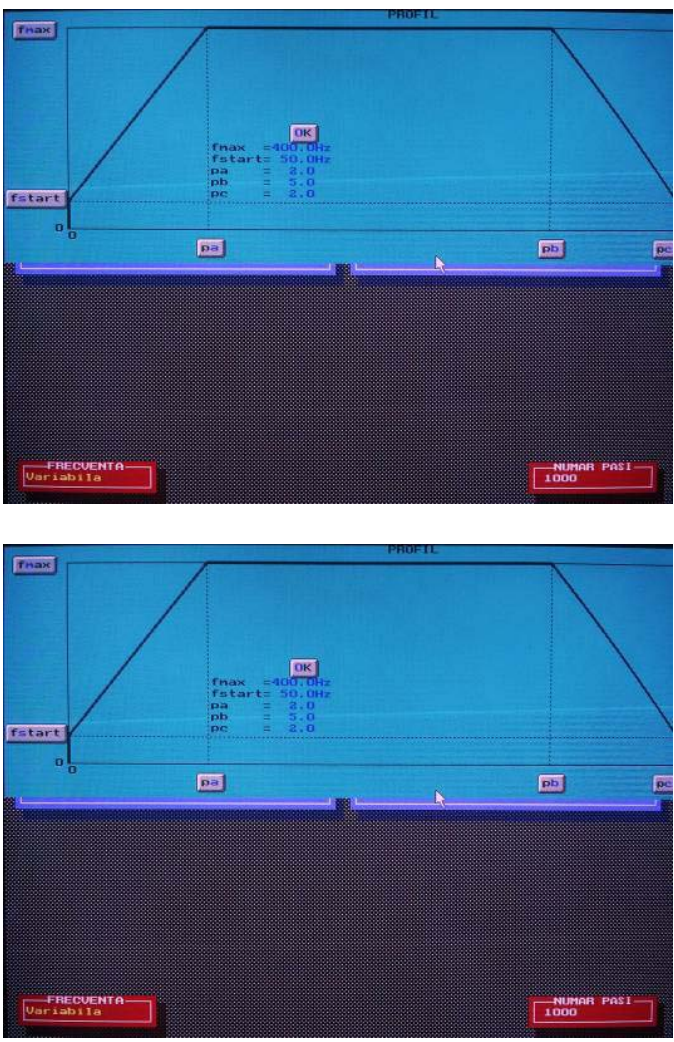

Fig. 15: Typical stepper acceleration and deceleration profile

\section{Conclusion}

This paper presents a design of a compact and intelligence five-phase stepping motor drive. With the special designed IC, the driver is smaller than a business card. Although it is a compact driver, it can provide a winding current and pulse rate up to 1.4 amperes and 60kpps, respectively. Self - test function also embedded inside the PCL-838 (80C31-CPU) of the driver so that engineers can check whether the driver is normal or not without an external equipment. Considering the positioning ability and precise speed control, this proposed driver can provide valuable Robotics and Automation Engineering applications involving either position control or speed control.

\section{References}

[1] Kenjo T, SugawaraA.(2003),Stepping Motors and Microprocessor Control, London, Oxford Clarendon Press.

[2] Acarnley PP.(1992),Stepping Motors: a Guide to Modern Theory and Practice, Peter Peregrinus Ltd., London.

[3] Takasaki K, Sugawara A.(1994),Stepping Motors and Their Microprocessor Controls, Clarendon Prsss, hbk, Oxford.

[4] Morar A.(2001),Sisteme electronice de comandă și alimentare a motoarelor pas cu pas implementate pe calculatoare pesonale (Electronic systems for stepping motor control implemented on personal computer) Ph. D. Thesis, Universitatea Tehnică din Cluj-Napoca.

[5] Morar A.(2007),Comanda inteligenta a actionarilor electrice cu motoare pas cu pas, Editura MEDIAMIRA, Cluj-Napoca.

[6] Botson M, Jeffrey SS, Stephen RS.(2006),Spontaneous Speed Reversals in Stepper Motor, IEEE Trans. Control Systems Technology, 14(2):pp. 369-373.

[7] McGuinessJ and Lahr B.(1994)Advantages of Five Phase Motors in Microstepping Drive, in Proc. IEE Colloquium on Stepper Motors and Their Control.

[8] Bellini A, Concari C, Franceschini G, Toscani A.(2007), Mixed-Mode PWM for Highperformance SteppingMotors, IEE Trans. Industrial Electronics, 54(6): pp. 3167-3177.

[9] Weerakoon TS, Samaranayake L.(2008),Development of a Novel Drive Topology for a Five Phase StepperMotor, In Proc. 2008 IEEE Industrial and Information Systems Conf., pp. 1-6.

[10]Adhikari P, Okaro M.(2008),Five-level Fivephase PWM Signal Generation using FPGA, IEE Trans. Industrial Electronics,,pp. 55(5).

[11]Farid B, Amar O.(2009),A Study of New Techniques of Controlled PWM Inverters, European Journal of Scientific Research, 32(1):pp. 77-87.

[12]Jong S-M, Siao S-T, Kang Y.(2010),An Implementation of FPGA Based Driver For approaching High Resolution of Five-phase Hibrid Stepper Motor,Machine Learning and Cybernetics (ICMLC), 2010 International Conference, 5: 2543-2548.

[13]Baluta G. (2004), Actionari electrice de mica putere-Aplicatii, Editura POLITEHNIUM, Iasi.

[14]***(2010), The Basics of Stepping Motors, Oriental Motor Co.Ltd, Japan.

[15]***(2010),Application Guides of Step Motor, Oriental Motor Co.Ltd, Japan.

[16]***(2012/20113),Oriental Motor-Stepping Motors-General Catalog.

[17]***(2010),STMicroelectronics, Motion Control Application Manual.

[18]***(2010),LEM Module, Data Book, Geneve, Switzerland.

[19]***(2011),Portescap: “Motion Systems".

[20]***(2008),PCL - 838 Stepping Motor Control Card, $P C-$ Lab Card Series Laboratory Manual. 